\title{
Antonio Ávila-Muñoz* and María Rodríguez Cruces* Estudio de las funciones de la atenuación en hablantes de Málaga con nivel de instrucción alto. Aproximación sociolingüística
}

\author{
Study on mitigation functions from a group of well-educated \\ Malaga speakers. A sociolinguistic approach
}

https://doi.org/10.1515/soprag-2020-0013

Published online octubre 30, 2020

\begin{abstract}
Mitigation is a pragmatic-linguistic category traditionally related to the theory of verbal politeness. Previous work on this issue has shown that conversational hedging is largely conditioned by the speaker's identity (Albelda 2012, Cestero 2011, Samper 2017), that is why a deeper understanding of attenuating phenomena requires close attention to the social context underpinning communicative interactions. This paper advances some preliminary results from the ongoing research on discursive mitigation in the Malaga-PRESEEA spoken corpus. The analysis is centered on the underlying mitigating functions in the speech performance of a group of speakers with university education. Since one of our main objectives is contrastive, and previous solid research on attenuation has been carried out within the PRESEEA context, its methodological basis has been assumed (Albelda 2012, Cestero 2011, Samper 2017). Results of multifactorial logistic regression analysis from our data are partially similar to those obtained in previous studies: mitigation formulas are frequent among the well-educated speakers we have studied, especially those related to self-protection functions. Women-more than men-, and younger speakers-more than every other age-group-, tend to lead this use.
\end{abstract}

\footnotetext{
*Corresponding authors: Antonio Ávila-Muñoz and María Rodríguez Cruces, Universidad de Málaga Facultad de Filosofia y Letras, Campus de Teatinos, Málaga, 29071, Spain, E-mail: amavila@uma.es. https://orcid.org/0000-0002-5239-2670
} 
Keywords: mitigation, linguistic variation, pragmatics, discourse analysis, PRESEEA

Resumen: La atenuación es una categoría pragmático-lingüística tradicionalmente relacionada con la teoría de la cortesía verbal. Trabajos anteriores demostraron que está condicionada, en gran medida, por los atributos sociales y personales de los hablantes, por lo que para entenderla plenamente es necesario atender a los factores sociales que la determinan (Albelda 2012, Cestero 2011, Samper 2017). Esta investigación presenta los resultados parciales del estudio de la atenuación discursiva en el corpus lingüístico PRESEEA-Málaga. Se analizan las funciones mitigadoras del discurso en un grupo de hablantes pertenecientes al nivel de instrucción alto. Contamos con investigaciones previas sobre la atenuación realizadas en el seno de PRESEEA-España, así que, para establecer comparaciones, asumimos los fundamentos metodológicos comunes a esos trabajos (Albelda 2012, Cestero 2011, Samper 2017). El análisis de regresión logística multifactorial que hemos realizado permite afirmar que nuestros resultados coinciden en parte con los presentados en estudios anteriores: las fórmulas de atenuación son frecuentes entre los informantes de nivel de instrucción alto que han sido estudiados en este trabajo. De las funciones analizadas, aquellas relacionadas con la autoprotección son las más frecuentes. En conjunto, las emplean más las mujeres que los hombres y los más jóvenes que los grupos de edad avanzado e intermedio.

Palabras clave: atenuación, variación lingüística, pragmática, análisis del discurso, PRESEEA

\section{Introducción}

La atenuación, en general, es un fenómeno que consiste en disminuir la tensión, la importancia o el valor de un hecho o de un acontecimiento. En el ámbito lingüístico, es una categoría pragmática que utilizan los hablantes para reducir la intensidad de lo que se dice o la carga negativa de ciertos mensajes y para formular explícitamente menos compromiso hacia lo que se expresa. A lo largo de la historia de los estudios sobre la atenuación lingüística, el concepto ha ido implementándose desde diferentes ámbitos y corrientes de investigación: pragmática, sociolingüística y análisis del discurso, principalmente. Los trabajos que abordan el fenómeno coinciden en que se trata de un recurso lingüístico asociado a las estrategias de cortesía comunicativa que se emplea para suavizar el discurso en busca de la aceptación del oyente o, en ocasiones, del propio hablante: con la intención de adecuar y adaptar los comportamientos lingüísticos individuales al 
discurso y a las reacciones de los interlocutores, los hablantes podrían desarrollar fórmulas de acercamiento comunicativo que involucren estrategias de atenuación.

Nuestro propósito es identificar y estudiar las principales funciones de la atenuación en 12 entrevistas semidirigidas pertenecientes al corpus oral del Proyecto para el Estudio Sociolingüístico del Español de España y América en Málaga (PRESEEA-Málaga). Por medio de un estudio estadístico queremos conocer cuáles son las características sociales que explican la variabilidad respecto al uso de las estrategias de la atenuación por parte de los informantes analizados atendiendo a las diferentes funciones que la literatura consultada le asigna a esta categoría pragmalingüística. Estas funciones se exponen en el apartado 2 de nuestro trabajo siguiendo la organización propuesta por Briz y Albelda (2013, p. 18).

El estudio cuantitativo realizado pretende, en última instancia, describir, analizar y explicar la existencia de estas funciones en el discurso de los informantes seleccionados y asociarlas a los grupos sociales en los que se integran según sus características personales. Los datos se han obtenido a través de la realización de entrevistas controladas mediante guion estructurado tal y como establece la metodología del Proyecto para el Estudio Sociolingüístico del Español de España y de América (Proyecto PRESEEA) coordinado por Francisco Moreno Fernández. Los 12 informantes analizados en nuestro trabajo pertenecen al grupo de nivel de estudios superior, con al menos tres años finalizados de formación universitaria. El análisis estadístico que hemos realizado de los datos consiste, por un lado, en una presentación descriptiva general y, por otro lado, en un estudio estadístico inferencial. El primero permite comprobar la dimensión de los datos usados; el segundo supone la realización una regresión lineal que estima o predice cómo la variación de los factores sociales considerados condiciona el comportamiento de la variable dependiente (funciones atenuadoras del discurso).

Las hipótesis planteadas en nuestra investigación sugieren que, debido a causas socioeducacionales bien conocidas desde hace tiempo que están relacionadas con el concepto de ‘conciencia sociolingüística' (López Morales 1989), las mujeres tenderán más que los hombres a usar las funciones que se derivan de la atenuación. Del mismo modo, asumimos que la edad es un factor condicionante en el empleo de las funciones de la atenuación: a menor edad, mayor empleo de estrategias de atenuación, pues está demostrado que las generaciones más jóvenes $-\mathrm{y}$ mejor instruidas- emplean con mayor frecuencia las estrategias generales de cortesía verbal (Bechara y Sánchez Gaviria 2012; Eckert 1997; Moreno Fernández 1998). Precisamente por esta razón, es probable que los más jóvenes hagan incluso mayor uso de las funciones menos utilizadas por todos los grupos etarios. El estilo de vida conservador de las personas de mayor edad (Ramos 2005, pp. 155-247), por su parte, las condicionará hacia el empleo más frecuente de las funciones que impliquen cierto grado de autoprotección que al resto de funciones consideradas en este 
trabajo. Igualmente, dado el carácter semiformal en el que se desarrollaron las entrevistas de las que se obtuvieron los datos de nuestro estudio, suponemos que las funciones analizadas que impliquen autoprotección serán las más empleadas de modo general. Este tipo de situación comunicativa fomenta el empleo de estrategias de atenuación mediante mecanismos de control, adecuación y adaptación del discurso. Las entrevistas semiformales se caracterizan por dinámicas discursivas gobernadas por procedimientos conscientes de acercamiento conversacional donde, no obstante, prevalece la salvaguarda del ego y de la imagen personal ante interlocutores desconocidos o poco conocidos (Albelda 2004).

\section{Marco teórico}

\subsection{Teoría de la atenuación}

El inicio del estudio propiamente dicho sobre la atenuación comunicativa se remonta a los años 70 con los trabajos pioneros de Lakoff (1973), Labov $(1966,1972)$ y Zadeh (1965). Más tarde, la tradición se consolidó con los estudios de Fraser (1980), Bravo (1993), Haverkate (2004), Briz (1995, 1998), Puga (1997), Ballesteros (2002), Albelda (2005, 2012), Caffi (2007), Albelda y Cestero (2011), Schneider (2013), Cestero (2011), entre otros.

Las investigaciones pronto repararon en el complejo mecanismo que supone la atenuación: por una parte, el hablante persigue un acercamiento o una aceptación social; por otra parte, ejecuta un alejamiento del mensaje. Este movimiento precisa de un equilibrio constante entre ambas fuerzas para lograr el éxito. Otras formas de acercamiento lingüístico se muestran más simples desde el momento en que, por ejemplo, los actos verbales valorizantes solo necesitan de la actualización de intensificadores discursivos (Briz 2007, p. 7). En el caso de la atenuación, sin embargo, el movimiento de alejamiento del mensaje (distanciamiento lingüístico) conlleva la necesidad de suavizarlo para conseguir el objetivo (acercamiento social).

Debido al mecanismo de acercamiento social que supone el fenómeno de la atenuación, este fue considerado desde sus orígenes como una estrategia propia de la cortesía comunicativa. Sin embargo, la cortesía no siempre explica completamente su uso ya que, a veces, incluso ni siquiera puede relacionarse de forma directa con la imagen del hablante (Cestero 2017, p. 59). La complejidad del acontecimiento exige entenderlo como un fenómeno pragmalingüístico dentro de las acciones consideradas de acercamiento y búsqueda de aceptación por parte de los interlocutores.

En análisis previos de la atenuación comunicativa, los factores que han demostrado tener mayor relevancia son, principalmente, tres: en primer lugar, la cantidad y el tipo de estrategias y recursos atenuadores que aparecen en un 
discurso; en segundo lugar, el contexto en el que se desarrollan las interacciones comunicativas, porque afecta a las estrategias usadas por los interlocutores; y, en tercer lugar, las diferentes posibilidades situacionales, porque pueden variar a lo largo del discurso y afectar a las señaladas estrategias de comunicación. Los aspectos lingüísticos, estructurales, enunciativos y situacionales que pueden servir de guía para reconocer cuándo un hablante persigue fines estratégicos atenuantes se han establecido en atención a las siguientes propiedades del acto lingüístico analizado: i) función general de la atenuación, ii) procedimientos de actuación, iii) factores estructurales en el análisis de la atenuación, iv) factores enunciativos y v) factores situacionales (Albelda et al. 2014, pp. 7-62).

En el presente estudio vamos a prestar atención únicamente a (i), las funciones generales de la atenuación, ya que estas han demostrado ser las más relevantes a la hora de analizar el fenómeno (Albelda et al. 2014, p. 16), no solo por su relevancia para entender dicha estrategia, sino también porque, al ser la propiedad más categórica de todas, su estudio nos permitirá obtener una visión general de su naturaleza. En síntesis, las funciones que vamos a estudiar en nuestro trabajo son: - Función 1: autoprotección. Los hablantes emplean esta función cuando pretenden velar por sí mismos evitando o reduciendo su compromiso con lo dicho.

Ejemplo 1. Sujeto 64

Entrevistador: ¿Cómo crees que viven los jóvenes la religión actualmente?

Entrevistada: Pufff.

Entrevistador: ¿En serio?

Entrevistada: Bueno / entiéndeme / yo creo que hay un (ruido) / jvamos! / nosotros lo estamos viendo / que de un tiempo a esta parte pues / muchos jóvenes se están acercando ¿no? (ruido) / hay algunos pues que no / o sea que están aquí pero no están / pero es lo típico ¿no? / pero que hay muchos jóvenes que se están acercando parece que buscando algo que no / que no encuentran en la sociedad / que no encuentran en su / en sus ambientes ¿no? / jvamos! / ahora de hecho hay // un grupo de diez jóvenes que han ido entrando / casi sin darte tú cuenta ¿no? / y jóvenes que buscan algo ¿no? // y algo que / ¡bueno! que no es lo que hay por ahí. Código de la entrevista: MA_064_M13 - Entrevista 819

En el ejemplo 1, se le ha preguntado a la entrevistada por los jóvenes y su relación con la religión. Los procedimientos atenuantes aparecen señalados en cursiva y muestran diversas estrategias que utiliza el hablante para no comprometerse totalmente con lo que está diciendo ante la solicitud de confirmación por parte del entrevistador (¿en serio?). Se observa un uso repetitivo de ¿no? -que llega a convertirse en muletilla discursiva - en un intento del hablante de buscar la aprobación e implicación de su interlocutor. De igual modo, la entrevistada rebaja $\mathrm{su}$ implicación tanto mediante fórmulas del tipo yo creo, parece que como mediante el empleo de diversas interjecciones (jvamos!, ¡bueno!). 
Ejemplo 2. Sujeto 736

Entrevistador: ¿Cómo está tu hermana Ángela?

Entrevistado: está mu(y) bien / mu(y) bien / la verdad es que está ibueno! / icon sus problemillas / que tiene también sus problemillas / por eso quiero estar con ella / más

Entrevistador: [uhum/]

Entrevistado: más que nada por apoyarla un poquito más de / porque yo creo que quieras que no / aunque tenga allí tres niños y el marido / pero se tiene que encontrar un poquito solilla y [...] Código de la entrevista: MA_736_H23 - Entrevista 959

En el ejemplo 2, al informante se le pregunta por su hermana y este responde autoprotegiendo la imagen de su familiar que, al parecer, está lejos del entorno que el informante considera más propicio para ella, a pesar de que se encuentra acompañada del marido y los hijos. La táctica lingüística atenuante más evidente en este fragmento es el empleo de los modificadores morfológicos internos: sufijos diminutivos.

- Función 2: prevención. Es una función atenuadora que se pone en marcha cuando el hablante intuye una posible amenaza a la imagen del otro o un posible obstáculo en la consecución de una meta. En este caso, se amplía la salvaguarda del ego hacia la proyección de la imagen del interlocutor.

Ejemplo 3. Sujeto 718

Entrevistador: ¿Tú qué harías si os tocara la lotería?

Entrevistado: si nos tocara muchísimo dinero // hay una cosa (risas) / que te puede parecer hasta tonta / esto que no conste en ningún sitio / tú ya me entiendes yo cuando paseo con Ginés muchas veces por el Paseo Marítimo / y veo cómo se está / desmoronando y descomponiendo / aquel antiguo / Hospital Dieciocho de julio, me gustaría echarlo abajo y reconstruirlo. Código de la entrevista: MA_718_M23 - Entrevista 1177

En el ejemplo 3, la entrevistada responde a una pregunta sobre sus planes de futuro en el hipotético caso de ser agraciada en un sorteo de lotería. Ella anticipa y previene su respuesta para minimizar la posibilidad de que su interlocutor pueda ofenderse con la opinión que expone sobre el estado del Hospital Dieciocho de julio de Málaga, cuya conservación y rehabilitación dependía en el momento de la entrevista del Ayuntamiento de la ciudad. El deterioro del histórico edificio supuso durante años causa de un profundo enfrentamiento político que se extendió al ámbito ciudadano.

Ejemplo 4. Sujeto 736

Entrevistador: Háblame ahora de / háblame ahora de tus padres por ejemplo / ¿cómo te llevas con ellos? 


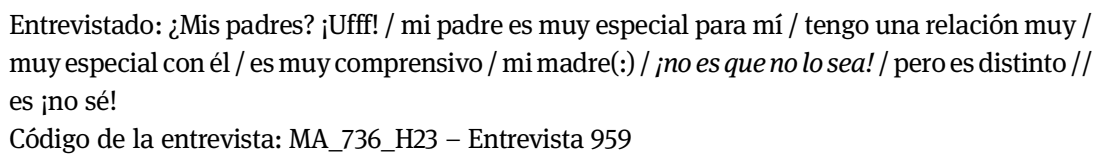

En el fragmento mostrado en el ejemplo 4, se le ha preguntado al entrevistado por la relación que mantiene con sus padres. Mientras se deshace en elogios hacia su padre, el entrevistado parece advertir que habla poco de su madre y emplea una estrategia de prevención para explicar cómo es su madre y aclarar que su relación con ella es buena: mi madre(:) / ino es que no lo sea! (especial, comprensiva).

- Función 3: reparación. Función usada para atenuar una amenaza a la imagen del otro o una intromisión en su territorio.

\section{Ejemplo 5. Sujeto 736}

[...] y a partir de ahí / ipues nada! / me metí en un despacho // que buscó mi hermana // de un compañero / además ese despacho me ha servi(d)o también para alegar en la / en la mili (sic) de que yo estaba trabajando / que me tenía que volver a Málaga / y no sé cuántos / ¡todo eso era más falso que judas! (risas) / ipero bueno! / ¡ya sabes! / iqué vamos a hacerle! Imagino que me entiendes, ¿no? Nadie quiere ir a la mili.

Código de la entrevista: MA_736_H23 - Entrevista 959

El ejemplo 5 muestra un fragmento donde se observa el empleo de la Función 3 (reparación) cuando el entrevistado ve que su imagen puede quedar dañada al confesar que mintió para no realizar el servicio militar. Se sirve de los elementos prosódicos y paralingüísticos, además de la reformulación expositiva y la búsqueda de implicación por parte del interlocutor (jya sabes!, imagino que me entiendes, ¿no?).

Ejemplo 6. Sujeto 148

Entrevistador: eso es // y en tu casa ¿cómo(:) / cómo lleváis la vida? / ¿quién hace las tareas la / de ca(:)sa? / ¿está repartida?

Entrevistada: ¡hombre! / por supuesto que no ¿no? / o sea / es lo típico ¿no? / o sea / tu madre(:) y las niñas ¿no? / o sea lo que es el padre y el niño ¡nada!

Entrevistador: nada ,

Entrevistada: jnada de nada!

Entrevistador:jnada de nada! / no colaboran en nada

Entrevistada: ¡nada de nada! / ¡hombre! algún que otro man / algún manda[d]illo o cosas así ¿no? / pero ¡qué va! /¡sí! / jahí va! / jeso!

Código de la entrevista: MA_148_M23 - Entrevista 3542

Por último, en el ejemplo 6, la entrevistada intenta reparar la mala imagen que ha declarado sobre su padre y sus hermanos al confesar que no participan en las 
labores de la casa y que todo el peso de esas actividades recae sobre la madre y las hermanas. La reparación consiste en asignarles al padre y a los hermanos ciertas tareas que ella considera menores al expresarlas en diminutivo (algún que otro manda(d)illo) o generalizarlas con la fórmula cosas así, al tiempo que intenta reconducir su discurso con una interjección (jhombre!).

\section{Metodología}

Generalmente, los estudios sobre la atenuación lingüística se han realizado a partir de la explotación de corpus orales obtenidos gracias a la investigación de comunidades de habla concretas. Esta es, sin duda, la mejor manera de estudiar este recurso, pues las características del código escrito facilitan que los hablantes puedan controlar su discurso de manera planificada y los recursos atenuantes son menos frecuentes y pueden resultar forzados y no naturales.

El PRESEEA fue diseñado por el profesor Moreno Fernández para describir, analizar y comprender la diversidad lingüística del español a partir de la recopilación y la explotación de corpus orales de las comunidades de habla de lengua española en todo el mundo. Los corpus son obtenidos a partir de entrevistas semidirigidas a través de un guion temático previo por medio del cual el entrevistador obtiene información diversa sobre el entrevistado. El papel del entrevistador debe ser simplemente el de estimular la participación de los informantes seleccionados y provocar, en la medida de lo posible, la aparición de diferentes tipos discursivos (diálogo, narración, descripción, argumentación). Junto a la realización de la entrevista, se le propone a los entrevistados cumplimentar un cuestionario en el que se recogen datos personales de cada uno de los informantes que componen la muestra. Cada uno de los grupos de investigación locales queda encargado de adaptar y desarrollar este cuestionario en función de las características particulares de la comunidad de habla estudiada y de los objetivos específicos de cada grupo de trabajo, aunque, de manera general, la información obtenida debe permitir conocer el nivel económico, la profesión, las condiciones de alojamiento y el modo de vida de los sujetos informantes.

Los subcorpus que integran la base de datos general de PRESEEA siempre deben estar constituidos por una muestra suficientemente amplia para que sea, por un lado, representativa del universo que se pretende analizar y, por otro lado, comparable con el resto de las muestras que se integran en el macrocorpus general. Esta es la razón por la que los grupos de investigación adscritos a PRESEEA trabajan con muestras por cuotas de afijación uniforme que dividen cada universo relativo en diferentes subpoblaciones atendiendo a las variables sociales género, edad y nivel de instrucción y asignan un número igual de informantes a cada uno 
Tabla 1: Muestra-tipo por cuotas propuesta para el Proyecto PRESEEA.

\begin{tabular}{|c|c|c|c|c|c|c|}
\hline & \multicolumn{2}{|c|}{$\begin{array}{l}\text { Generación } 1 \\
\text { (20-34 años) }\end{array}$} & \multicolumn{2}{|c|}{$\begin{array}{l}\text { Generación } 2 \\
\text { (35-55 años) }\end{array}$} & \multicolumn{2}{|c|}{$\begin{array}{c}\text { Generación } 3 \\
\text { (56 años o más) }\end{array}$} \\
\hline & Hombres & Mujeres & Hombres & Mujeres & Hombres & Mujeres \\
\hline $\begin{array}{l}\text { Nivel de estudios } \\
1 \text { (analfabetos } \\
\text { o primaria) }\end{array}$ & & & & & & \\
\hline $\begin{array}{l}\text { Nivel de estudios } \\
2 \text { (secundaria) }\end{array}$ & & & & & & \\
\hline $\begin{array}{l}\text { Nivel de estudios } \\
3 \text { (estudios } \\
\text { superiores) }\end{array}$ & & & & & & \\
\hline
\end{tabular}

de esos grupos sociales. La Tabla 1 representa la muestra-tipo del Proyecto PRESEEA.

Para el tamaño de cada muestra se propone una asignación uniforme de cuatro informantes por cada una de las casillas mostradas en la Tabla 1, lo que supone un total de 72 informantes por cada una de las muestras y plantea una representatividad de 1/25000 hablantes para ciudades de alrededor de dos millones de habitantes, aunque se prevé mucho más holgada para aquellos núcleos urbanos de menor población.

A partir del estudio de la base de datos generada en el seno de PRESEEA, y gracias al diseño de metodologías comunes que permiten comparaciones y estudios coordinados, diversas investigaciones han realizado trabajos sociolingüísticos sobre la variación lingüística, variación fonético-fonológica, variación sintáctica, variación léxica, variación en el discurso, etnografía de la comunicación, sociología del lenguaje, pragmática, etcétera. Marta Albelda, Marta Samper y Ana $\mathrm{M}^{\mathrm{a}}$ Cestero han sido pioneras a la hora de analizar la atenuación dentro del corpus oral de PRESEEA. En sus trabajos examinan algunas entrevistas realizadas en sus respectivas zonas de origen (Las Palmas de Gran Canaria (Samper 2013), Madrid-Alcalá (Cestero 2015, 2017), Valencia (Albelda 2012)). Se trata de estudios en los que se analizan los recursos atenuantes y los actos del habla con atenuación de muestras de informantes estratificadas según las directrices señaladas propias del Proyecto PRESEEA.

La ciudad de Málaga cuenta en la actualidad con cerca de 600 mil habitantes y el Proyecto PRESEEA-Málaga respeta la cuota uniforme fijada en las indicaciones del macroproyecto. Se han considerado cuatro informantes para cada una de las celdas resultantes de la muestra, lo que supone para esta ciudad una representación de un informante por cada 7746 habitantes. 
Tabla 2: Muestra analizada. Nivel de instrucción alto.

\begin{tabular}{lll}
\hline Edad & Género \\
\hline Grupo 1 (18-35 años) & Mujer $(N=2)$ & Hombre $(N=2)$ \\
Grupo 2 (36-55 años) & Mujer $(N=2)$ & Hombre $(N=2)$ \\
Grupo 3 (más de 55 años) & Mujer $(N=2)$ & Hombre $(N=2)$ \\
\hline
\end{tabular}

En concreto, la investigación que mostramos aquí se ha realizado a partir del estudio de textos obtenidos de las entrevistas a informantes de nivel de estudios superior y, en concreto, de una submuestra de 12 entrevistas seleccionadas aleatoriamente ( $N=12$; seis mujeres y seis hombres). Se establecieron tres grupos de edad (Grupo 1: 18-35 años; Grupo 2: 36-55 años; Grupo 3: más de 55 años). Aunque esta división etaria difiere ligeramente de la presentada en la Tabla 1 referida a las directrices generales de PRESEEA, ha de tenerse en cuenta que en las propias normas se advierte que los criterios comunes constituyen un mínimo metodológico que persigue la comparabilidad de materiales, aunque las circunstancias locales permiten a los investigadores adaptar las bases de uso común a la realidad estudiada. Por esta razón, los grupos etarios de nuestra muestra se han adaptado a la situación académica española que establece el inicio de la etapa universitaria alrededor de los 18 años.

En esquema, la distribución de la muestra analizada en este trabajo puede observarse en la Tabla 2.

Una vez seleccionadas las entrevistas, se identificaron las tres funciones de la atenuación expuestas en el apartado 2.1 de este estudio. Aunque, por motivos de comparación, la muestra se estratificó desde el inicio siguiendo el diseño de los trabajos previos realizados en el seno de PRESEEA (género, edad), en principio se tuvieron en cuenta todos los atributos personales y sociales de los informantes analizados, así como el tipo de relación existente entre los participantes en las entrevistas (relación entrevistador/entrevistado). Los atributos personales señalados se obtuvieron gracias a los cuestionarios sociológicos complementarios usados de forma habitual en PRESEEA-Málaga. Estos cuestionarios facilitan el acceso a un considerable número de variables de pre- y postestratificación que atienden a las características particulares de los informantes y que, por tanto, podrían tener valor explicativo de la variación lingüística. Dichos cuestionarios eran completados por los informantes una vez que finalizaban las entrevistas y proporcionan información relativa a tres bloques de variables: sociales, psicosociales y reticulares. Las variables sociales se refieren a los datos personales de los hablantes; las variables psicosociales permiten la construcción de escalas que miden la integración de los 
sujetos en la cultura de corriente dominante y su integración en la cultura local; las variables reticulares hacen referencia a las relaciones de los entrevistados con su red social personal. Todas ellas han sido usadas de forma fructífera en el desarrollo del Proyecto PRESEEA-Málaga, por lo que el lector interesado puede encontrar información complementaria sobre los detalles de estas variables en cualquiera de los trabajos publicados al respecto (Lasarte et al. 2008; Lasarte y Villena 2008; Vida 2007).

\section{Análisis de los resultados}

En este apartado vamos a realizar una presentación estadístico-descriptiva general de los datos y un análisis inferencial que consistirá en la realización de una regresión lineal con el propósito de estimar o predecir el comportamiento de la variable dependiente (funciones atenuadoras del discurso) a través de la variación de los factores sociales que, tras el análisis de todos los contenidos en los cuestionarios citados, mostraron tener mayor relevancia en la explicación del fenómeno: género y edad de los informantes. Se han realizado dos tipos de regresión:

- Regresión lineal simple entre cada una de las funciones generales de la atenuación con las variables explicativas por separado. Con este análisis se pretende explicar cómo influyen el género y la edad en las funciones generales de la atenuación, individualmente.

- Regresión lineal múltiple como extensión de la regresión simple. Se realiza entre la variable dependiente y la combinación de las variables independientes que resultaron significativas (género y edad). El objetivo es estudiar de qué manera influyen los predictores en conjunto sobre la variable endógena (funciones atenuantes) y, también, observar qué relación hay entre las variables exógenas (género y edad).

\subsection{Análisis descriptivo general}

Las 12 entrevistas analizadas suponen el estudio de diez horas y cuarenta y cinco minutos de grabación en formato digital. Aunque, como hemos señalado, las entrevistas fueron previamente planificadas mediante un guion estructurado, la tarea principal consistió en que el informante sintiera la comodidad suficiente como para emplear progresivamente los estilos más espontáneos y los recursos asociados a ellos. Por este motivo, la mayor parte de las grabaciones se realizaron en los domicilios de los entrevistados o en lugares que ellos proponían, siempre 
Tabla 3: Datos generales del uso de las funciones de la atenuación en el corpus PRESEEA-Málaga.

\begin{tabular}{lrrrrrr}
\hline Código del informante & \multicolumn{3}{c}{ Función general de la atenuación } & $N$ & Género & Edad \\
\cline { 2 - 5 } & $\begin{array}{l}\text { Autoprotección } \\
\text { Función 1 }\end{array}$ & $\begin{array}{r}\text { Prevención } \\
\text { Función 2 }\end{array}$ & $\begin{array}{r}\text { Reparación } \\
\text { Función 3 }\end{array}$ & & & \\
\hline Informante 005 & 57 & 7 & 38 & 102 & 1 & 1 \\
Informante 732 & 115 & 18 & 61 & 194 & 1 & 1 \\
Informante 736 & 81 & 10 & 26 & 117 & 1 & 2 \\
Informante 791 & 43 & 8 & 12 & 63 & 1 & 2 \\
Informante 714 & 124 & 6 & 15 & 145 & 1 & 3 \\
Informante 725 & 109 & 5 & 22 & 136 & 1 & 3 \\
Informante 64 & 85 & 9 & 25 & 119 & 0 & 1 \\
Informante 148 & 138 & 16 & 20 & 174 & 0 & 1 \\
Informante 718 & 91 & 11 & 11 & 113 & 0 & 2 \\
Informante 711 & 86 & 36 & 17 & 139 & 0 & 2 \\
Informante 720 & 84 & 40 & 19 & 143 & 0 & 3 \\
Informante 706 & 94 & 29 & 16 & 139 & 0 & 3 \\
& 1107 & 195 & 282 & 1584 & & \\
\hline
\end{tabular}

Género: 0 = Mujer; 1 = Hombre Edad: 1 = Grupo 1 (18-35 años); 2 = Grupo 2 (36-55 años); 3 = Grupo 3 (más de 55 años).

que se respetasen unos niveles mínimos de calidad acústica, ya que los materiales se emplearon también para realizar estudios fonéticos dentro del ámbito general de PRESEEA-Málaga. Del total de grabaciones analizadas, se han obtenido y

Tabla 4: Porcentaje individual de uso de funciones. Datos particulares.

\begin{tabular}{lrrr}
\hline $\begin{array}{l}\text { Código del } \\
\text { informante }\end{array}$ & $\begin{array}{r}\text { Autoprotección } \\
\text { Función 1 }\end{array}$ & $\begin{array}{r}\text { Prevención } \\
\text { Función 2 }\end{array}$ & $\begin{array}{r}\text { Reparación } \\
\text { Función 3 }\end{array}$ \\
\hline Informante 005 & 55,9 & 6,9 & 37,2 \\
Informante 732 & 59,3 & 9,3 & 31,4 \\
Informante 736 & 69,2 & 8,5 & 22,2 \\
Informante 791 & 68,3 & 12,7 & 19 \\
Informante 714 & 85,6 & 4,1 & 10,3 \\
Informante 725 & 80,1 & 3,7 & 16,2 \\
Informante 64 & 71,4 & 7,6 & 21 \\
Informante 148 & 79,3 & 9,2 & 11,5 \\
Informante 718 & 80,6 & 9,7 & 9,7 \\
Informante 711 & 61,9 & 25,9 & 12,2 \\
Informante 720 & 58,7 & 28 & 13,3 \\
Informante 706 & 67,6 & 20,9 & 11,5 \\
\hline
\end{tabular}




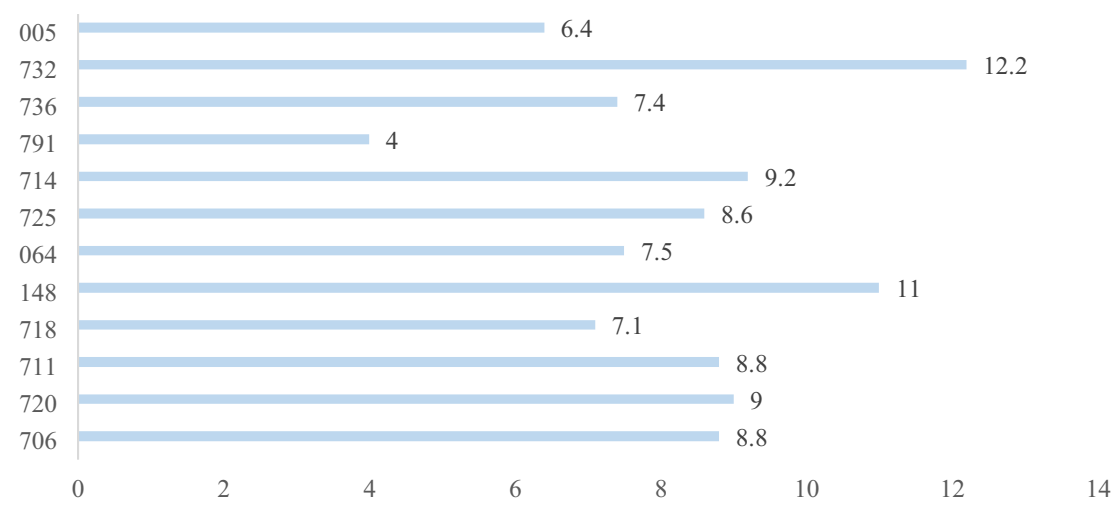

Gráfico 1: Porcentaje individual de uso de funciones. Datos generales.

estudiado 1584 casos de las funciones de la atenuación. En la Tabla 3 se presenta su distribución entre la muestra.

La distribución de los 1584 casos observados corresponde a 1107 ejemplos de la función de autoprotección (Función 1, 69,9 \%), 195 de prevención (Función 2, 12,3%) y 282 de reparación (Función 3, 17,8 \%). Destacan, como puede observarse en la Tabla 3, los casos de la función de autoprotección (Función 1) que abarcan casi el $70 \%$ del total $(N=1107$ casos sobre un total de 1584).

Es significativo que todos los sujetos usan más de 100 casos relacionados con las funciones de la atenuación, a excepción del identificado con el número 791 que solo empleó 63. El entrevistado 732 es el que más casos de funciones utiliza para atenuar su discurso $(N=194)$. La función más veces empleada por un solo sujeto ha sido la de autoprotección (Sujeto 148, $N=138$ ); y la que menos veces ha sido usada por un solo individuo fue la prevención (Sujeto 725, $N=5$ ).

La Tabla 4 y el Gráfico 1 muestran, respectivamente, el porcentaje de los casos de funciones particulares (Tabla 4) y generales (Gráfico 1) de atenuación utilizadas por cada uno de los sujetos estudiados.

La Tabla 4 corrobora que la autoprotección (Función 1) es la función que usan más todos los entrevistados. Estos valores son mucho mayores, con diferencia, que el resto de resultados obtenidos en las otras dos funciones.

Si tenemos en cuenta las características sociales de los hablantes analizados (véase Tabla 3), se pueden extraer del Gráfico 1 ciertas afirmaciones relacionadas con el género y la edad de la muestra analizada que, posteriormente, serán relevantes en el análisis de regresión: 
1. El entrevistado que más casos de funciones utiliza es un hombre (Informante 732) con un 12,2 \% del total de casos computados; el segundo porcentaje más alto pertenece a una mujer (Informante 148) con un $11 \%$ del total de casos analizados.

2. Los entrevistados pertenecientes al Grupo 3 (más de 55 años; informantes 714 , 725, 720 y 706) presentan unos valores semejantes: todos oscilan entre el 8,8 \% y 9,2\% sobre el total de casos obtenidos para la muestra completa.

3. Los dos porcentajes más altos en cuanto al empleo de casos de funciones (informantes 732 y 148) corresponden a dos entrevistados del Grupo 1 (18-36 años).

4. Las mujeres de la muestra presentan porcentajes considerablemente similares en cuanto al empleo de casos de las funciones analizadas; estos porcentajes son más dispares en los hombres.

\subsubsection{Análisis bivariante}

Como hemos señalado más arriba, las dos variables que manifestaron un poder de explicación más elevado sobre la variable dependiente y mostraron unos niveles de correlación estadísticamente más significativos fueron el género del informante y su edad.

\subsubsection{Género}

En la Tabla 5 se muestran los resultados porcentuales y absolutos de casos de las funciones generales de la atenuación según el género de las personas analizadas:

Tabla 5: Resultados generales según el género de los informantes.

\begin{tabular}{|c|c|c|c|c|c|}
\hline Género & $\begin{array}{r}\text { Autoprotección } \\
\text { Función } 1\end{array}$ & & $\begin{array}{r}\text { Prevención } \\
\text { Función } 2\end{array}$ & $\begin{array}{r}\text { Reparación } \\
\text { Función } 3\end{array}$ & \\
\hline \multirow[t]{2}{*}{ Hombre } & 529 & & 54 & 174 & 757 \\
\hline & $33,40 \%$ & & $3,41 \%$ & $10,98 \%$ & $47,79 \%$ \\
\hline \multirow[t]{2}{*}{ Mujer } & 578 & & 141 & 108 & 827 \\
\hline & $36,50 \%$ & & $8,90 \%$ & $6,82 \%$ & $52,21 \%$ \\
\hline \multirow[t]{2}{*}{ Total } & 1107 & & 195 & 282 & 1584 \\
\hline & $69,89 \%$ & & $12,31 \%$ & $17,80 \%$ & $100 \%$ \\
\hline Prueba & Estadístico & GI & Valor-P & & \\
\hline Chi-Cuadrado & 53,442 & 2 & 0,0000 & & \\
\hline
\end{tabular}


Las mujeres hacen uso de las funciones atenuantes en más ocasiones que los hombres. Aunque se observa que la diferencia de utilización de los casos de las funciones generales de la atenuación entre ambos géneros es baja $(\mathrm{H}=47,79 \%$; $M=52,21 \%$ ), esta diferencia resultó ser significativa $\chi^{2}$ (Sig. 0,00000).

La autoprotección (Función 1) es la más usada por ambos géneros. Cabe señalar que esta función es la que se emplea con menor diferencia entre ambos grupos ( $\mathrm{H}=33,4 \% ; \mathrm{M}=36,5 \%$ ). La prevención (Función 2) es la menos utilizada por los hombres $(3,41 \%)$ y la reparación (Función 3), la menos usada por las mujeres $(6,82 \%)$.

\subsubsection{Edad}

La Tabla 6 muestra la correlación de las funciones de la atenuación y la edad.

Tabla 6: Resultados generales según la edad de los informantes.

\begin{tabular}{|c|c|c|c|c|}
\hline Edad & $\begin{array}{r}\text { Autoprotección } \\
\text { Función } 1\end{array}$ & $\begin{array}{r}\text { Prevención } \\
\text { Función } 2\end{array}$ & $\begin{array}{r}\text { Reparación } \\
\text { Función } 3\end{array}$ & \\
\hline \multirow[t]{2}{*}{ Grupo 1 (18-35 años) } & 395 & 50 & 144 & 589 \\
\hline & $24,95 \%$ & $3,16 \%$ & $9,09 \%$ & $37,18 \%$ \\
\hline \multirow[t]{2}{*}{ Grupo 2 (36-55 años) } & 301 & 65 & 66 & 432 \\
\hline & $19 \%$ & $4,10 \%$ & $4,17 \%$ & $27,27 \%$ \\
\hline \multirow[t]{2}{*}{ Grupo 3 (mayores de 55 años) } & 411 & 80 & 72 & 563 \\
\hline & $25,95 \%$ & $5,05 \%$ & $4,55 \%$ & $35,54 \%$ \\
\hline \multirow[t]{2}{*}{ Total } & 1107 & 851 & 282 & 1584 \\
\hline & $69,89 \%$ & $12,31 \%$ & $17,80 \%$ & $100 \%$ \\
\hline Estadístico & Gl & Valor-P & & \\
\hline Chi-Cuadrado & 28 & 0,0000 & & \\
\hline
\end{tabular}

De nuevo, existe una correlación significativa entre las funciones de la atenuación y el factor edad (Sig. = 0,0000). El Grupo 1 (18-35 años) es el que más casos de funciones emplea para atenuar el discurso $(N=589,37,18 \%)$, seguido del Grupo 3 (mayores de 55 años) $(N=563,35,54 \%)$ y, por último, el grupo de edad intermedio (36-55 años) $(N=432,27,27 \%)$.

Los tres grupos tienen unos valores absolutos relativamente bajos en la prevención (Función 2). Estos oscilan entre 50 y 80 repeticiones, aunque los miembros del Grupo 3 (mayores de 55 años) son los que más emplean esta función. Además, la distribución progresiva ascendente de los valores indica que, a mayor edad, mayor empleo de la función de prevención. 
La autoprotección (Función 1) es la más usada por todos los grupos etarios, mientras que las funciones menos empleadas son prevención (Función 2) en el Grupo 1 (18-35 años) y Grupo 2 (36-55 años), y reparación (Función 3), en el Grupo 3 (mayores de 55 años). Se observa que el empleo de la prevención y reparación en el grupo etario intermedio es similar, los resultados muestran una sola repetición de diferencia ( $N=65$ en prevención; $N=66$ en reparación).

\subsubsection{Análisis de regresión lineal}

Para medir la influencia de las variables que se mostraron más influyentes en cada una de las funciones generales de la atenuación, se han efectuado tres análisis de regresión lineal, uno por cada función. La Tabla 7 muestra los resultados generales obtenidos:

Tabla 7: Análisis de regresión lineal.

\begin{tabular}{|c|c|c|c|c|c|c|c|c|}
\hline \multicolumn{3}{|c|}{ Función 1} & \multicolumn{3}{|c|}{ Función 2} & \multicolumn{3}{|c|}{ Función 3} \\
\hline Edad & $\begin{array}{l}\text { Mult. } \\
R \text {-squared }\end{array}$ & 0,004012 & Edad & $\begin{array}{l}\text { Mult. } \\
R \text {-squared }\end{array}$ & 0,06926 & Edad & $\begin{array}{l}\text { Mult. } \\
R \text {-squared }\end{array}$ & 0,3058 \\
\hline & $p$-value & 0,8438 & & $p$-value & 0,4085 & & $p$-value & 0,0622 \\
\hline \multirow[t]{2}{*}{ Género } & $\begin{array}{l}\text { Mult. } \\
R \text {-squared }\end{array}$ & 0,02546 & Género & $\begin{array}{l}\text { Mult. } \\
R \text {-squared }\end{array}$ & 0,3883 & Género & $\begin{array}{l}\text { Mult. } \\
R \text {-squared }\end{array}$ & 0,1713 \\
\hline & $p$-value & 0,6203 & & $p$-value & 0,0304 & & $p$-value & 0,181 \\
\hline $\begin{array}{c}\text { Edad } \\
+\end{array}$ & $\begin{array}{l}\text { Mult. } \\
\text { R-squared }\end{array}$ & 0,02953 & $\begin{array}{l}\text { Edad } \\
+\end{array}$ & $\begin{array}{l}\text { Mult. } \\
R \text {-squared }\end{array}$ & 0,4576 & $\begin{array}{l}\text { Edad } \\
+\end{array}$ & $\begin{array}{l}\text { Mult. } \\
R \text {-squared }\end{array}$ & 0,4771 \\
\hline Género & $p$-value & 0,8732 & Género & $p$-value & 0,06375 & Género & $p$-value & 0,05406 \\
\hline
\end{tabular}

alpha $=0,05$. Predicciones fiables solo si el coeficiente de determinación $>0,7$.

A la vista de la Tabla 7, podríamos obtener dos conclusiones, principalmente:

I) Por un lado, el factor género es significativo en la prevención (Función 2), con un $p$-valor de 0,0304. Este valor apunta a que existe una relación lineal entre las variables estudiadas.

II) En cuanto al nivel de bondad de ajuste, la predicción no se mostró fiable, ya que el coeficiente de determinación es menor a 0,7 . Por último, mostramos en la Tabla 8 los coeficientes de la regresión correspondiente en el caso donde se ha rechazado la hipótesis nula.

\section{Discusión y conclusiones}

Tras el análisis expuesto, hemos comprobado que los hablantes de la ciudad de Málaga con un nivel de estudios alto emplean de manera frecuente estrategias 
Tabla 8: Coeficientes de la regresión lineal simple.

\begin{tabular}{lrrrr}
\hline \multicolumn{5}{c}{ Prevención } \\
\hline \multicolumn{5}{c}{ Función 2 } \\
\hline Coeficiente & Estimador & $\begin{array}{c}\text { Error } \\
\text { estándar }\end{array}$ & Valor t & $\operatorname{Pr}(>t)$ \\
\hline Intercepto & & 4,069 & 5,775 & 0,000179 \\
Género & 23,5 & 5,755 & $-2,52$ & 0,030404 \\
\hline
\end{tabular}

En la Tabla 8 se observa que el coeficiente de la variable género en la Función 2 es negativo, lo que nos indica que la variable dependiente cambiará de modo inverso con respecto al crecimiento 0 decrecimiento de la variable independiente $($ Mujer $=0$; Hombre $=1)$.

de atenuación discursiva para buscar el acercamiento a sus interlocutores a partir de la situación comunicativa generada en el contexto de entrevista semiformal propia de la metodología de obtención de datos que caracteriza al proyecto PRESEEA. La autoprotección (Función 1) es la función atenuadora empleada de manera mayoritaria, mientras que la prevención (Función 2) y la reparación (Función 3) son empleadas, prácticamente, con la misma frecuencia.

Los resultados estadísticos en relación al género muestran que las mujeres utilizan más las funciones generales de la atenuación que los hombres. La autoprotección (Función 1) es la más usada por los dos grupos. La que menos emplean los hombres es la prevención (Función 2), mientras que la reparación (Función 3) es la menos utilizada por las mujeres.

Respecto a la edad, son los más jóvenes de nuestro estudio los que más casos de funciones muestran, seguidos del grupo de mayor edad y, por último, el grupo etario intermedio, que es el que menos casos de funciones atenuadoras manifiesta. Se puede afirmar también que, de nuevo, la autoprotección (Función 1) es la función más veces empleada en los tres grupos; la prevención (Función 2), la menos usada por los más jóvenes (18-35 años) y el grupo de edad intermedio (36-55 años) y la reparación (Función 3), por las personas de edad más avanzada.

Los análisis realizados nos permiten afirmar, asimismo, que las dos variables sociales consideradas y las tres funciones de la atenuación analizadas están relacionadas estadísticamente y tienen dependencia entre sí. De igual modo, se ha demostrado que en el único caso donde alguna variable es significativa está involucrado el género. Esta variable explica las variaciones en la prevención (Función 2). No obstante, hemos de ser cautos con la posible interpretación que se derive de esta covariación, ya que los coeficientes de determinación no alcanzan el valor mínimo preestablecido y, por lo tanto, necesitaremos en el futuro ampliar la muestra para consolidar estos primeros hallazgos. 
A pesar de ello, y a la vista de los resultados obtenidos hasta ahora, pueden establecerse algunas comparaciones parciales con los trabajos ya realizados en el seno de PRESEEA-España relacionados con las fórmulas de atenuación (Albelda 2012; Cestero 2011, 2015, 2017; Samper 2013, 2017).

A) En todos los trabajos anteriores se observan diferencias entre mujeres y hombres en los casos donde se ponen de manifiesto las funciones de la atenuación. Aunque estas no son muy significativas en ninguno de los estudios analizados, lo cierto es que, en las investigaciones llevadas a cabo en Madrid (Cestero 2011) y Las Palmas (Samper 2017), los hombres atenuaban más que las mujeres, si bien la significación obtenida no era muy alta y, por tanto, coincide en lo esencial con la que nosotros hemos encontrado en Málaga.

B) En los estudios previos, los más jóvenes atenúan más que los más mayores, lo que vuelve a corroborarse en nuestro trabajo. El grupo etario intermedio muestra comportamientos diferentes en las investigaciones que nos sirven de referencia: en Las Palmas, este grupo ocupa una posición media en cuanto al empleo de estrategias atenuadoras, mientras que en Madrid y Valencia resultó ser el grupo de edad que más atenuaba; en nuestro caso, por el contrario, se ha mostrado como el grupo que manifiesta menor porcentaje de uso de las funciones atenuadoras.

En definitiva, nuestras conclusiones indican que los malagueños de nivel de instrucción alto utilizan con frecuencia fórmulas de atenuación, en especial aquellas relacionadas con la autoprotección. Lo hacen más las mujeres que los hombres, en una manifestación más de su mayor conciencia sociolingüística (López Morales 1989), y los más jóvenes que los grupos de edad avanzado e intermedio, lo que supone consolidar la idea de que los jóvenes más instruidos manifiestan con frecuencia estrategias generales de atenuación y cortesía verbal (Eckert 1997; Moreno Fernández 1998; Bechara y Sánchez Gaviria 2012).

El uso frecuente por parte de la población general con estudios superiores de funciones atenuadoras puede interpretarse como el efecto de una intención consciente por parte de los hablantes de adaptar sus intervenciones a las de sus interlocutores. Se trataría, en definitiva, de buscar un acercamiento comunicativo que les haga sentir más seguros a lo largo de la situación de entrevista semiformal que genera las interacciones analizadas.

Es probable que, como ocurre en trabajos realizados en otras zonas (Cestero 2017), los entrevistados con nivel superior de estudios atenúen el discurso en más ocasiones que los del grupo de instrucción medio o básico. Esperamos poder corroborar esta y otras conjeturas pergeñadas en este trabajo en futuras investigaciones, cuando se amplíe la muestra y se analicen sujetos de los tres grupos de educación contemplados en el diseño del Proyecto PRESEEA-Málaga. 
Agradecimientos: Este trabajo ha sido posible gracias a la explotación de la base de datos lingüísticos que desde hace más de treinta años viene compilando en la Universidad de Málaga el Proyecto de Estudio Sociolingüístico del Español de España y de América en Málaga (PRESEEA-Málaga. Códigos de referencia DGICyT FFI2011-29189-C05-01, FFI2015-68171-C5-1-P, PID2019-104982GB-C5-2). Agradecemos a su coordinador, el Profesor Juan Andrés Villena Ponsoda, su apoyo, tanto por facilitarnos el acceso a los datos, como por el asesoramiento desinteresado en las diferentes etapas de este artículo, en especial, aquellas referidas a los análisis estadísticos de los datos.

\section{Referencias}

Albelda, M. (2004). Cortesía en diferentes situaciones comunicativas. La conversación coloquial y la entrevista sociológica semiformal. In D. En Bravo \& A. Briz (coord.), Pragmática sociocultural: estudios sobre el discurso de cortesía en español (pp. 109-136). Madrid: Ariel. Albelda, M. (2005). La intensificación en el español coloquial. Valencia: Universitat.

Albelda, M. (2012). Estudio sociolingüístico (piloto) de la atenuación en el corpus PRESEEA de Valencia. In E. En Ridruejo Alonso (coord), Tradición y progreso en la Lingüística General (pp. 7-28). Valladolid: Universidad.

Albelda, M., Briz, A., Cestero, A. M., Kotwica, D., \& Villalba, C. (2014). Ficha metodológica para el análisis pragmático de la atenuación en corpus discursivos del español. Oralia, 17, 7-62.

Albelda, M. \& Cestero, A. M. (2011). De nuevo, sobre los procedimientos de atenuación lingüística. Espanol Actual, 96, 121-155.

Ballesteros, F. J (2002). Mecanismos de atenuación en español e inglés. Implicaciones pragmáticas de la cortesía. Círculo de Lingüística Aplicada a la Comunicación, 11. Publicación en línea. [Consulta, 13 de junio de 2020].

Bechara, O. \& Sánchez Gaviria, D. C. (2012). Estrategias de cortesía que utilizan los jóvenes para demostrar solidaridad cuando aconsejan [Tesis de Licenciatura], Pontificia Universidad Javeriana de Bogotá.

Bravo, D. (1993). La atenuación de las divergencias mediante la risa en negociaciones españolas y suecas. Estocolmo: Publicaciones de la Universidad de Estocolmo.

Briz, A. (1995). La atenuación en la conversación coloquial. Una categoría pragmática. In L. En Cortés (Ed), El español coloquial: actas del I Simposio sobre análisis del discurso oral (pp. 103-122). Almería: Universidad de Almería.

Briz, A. (1998). El español coloquial. Esbozo de pragmagramática. Barcelona: Ariel.

Briz, A. (2007). Para un análisis semántico, pragmático y sociopragmático de la cortesía atenuadora en España y América. Linguistica Española Actual, 29(1), 5-40.

Briz, A. \& Albelda, M. (2013). Una propuesta teórica y metodológica para el análisis de la atenuación lingüística en español y portugués. La base de un proyecto (ES.POR.ATENUACIÓN). Onomázein: Revista de lingüística, filología y tradución de la Pontificia Universidad Católica de Chile, 28, 288-319.

Caffi, C. (2007). Mitigation. Oxford: Elsevier. 
Cestero, A. M. (2011). Estudio sociolingüístico de la atenuación en el corpus PRESEEA-Madrid. In A. M. Cestero, I. Molina, \& F. Paredes (Eds), La lengua, lugar de encuentro. Actas del XVI Congreso Internacional de la Alfal (pp. 1897-1906). Alcalá de Henares: Servicio de Publicaciones de la Universidad de Alcalá.

Cestero, A. M. (2015). La atenuación lingüística en el habla de Madrid: Un fenómeno sociopragmático variable. In A. M. Cestero, I. Molina, \& F. Paredes (eds.), Patrones sociolingüísticos de Madrid (pp. 365-412). Berna: Peter Lang.

Cestero, A. M. (2017). La atenuación en el habla de Madrid: Patrones sociopragmáticos. Rilce. Revista de Filología Hispánica, 33(1), 57-86.

Eckert, P. (1997). Age as a sociolinguistic variable. In F. En Coulmas (Ed.), The handbook of sociolinguistics (pp. 151-167). Oxford: Oxford University Press.

Fraser, B. (1980). Conversational mitigation. Journal of Pragmatics, 4, 341-350.

Haverkate, H. (2004). El análisis de la cortesía comunicativa, categorización pragmalingüística de la cultura española. In D. En Bravo \& A. Briz (Eds.), Pragmática sociocultural: Estudios sobre el discurso de cortesía en español (pp. 55-65). Barcelona: Ariel.

Labov, W. (1966). The social stratification of English in New York city. Cambridge: Cambridge University Press.

Labov, W. (1972). Sociolinguistic patterns. Filadelfia: University of Pennsylvania Press.

Lakoff, R. (1973). The logic of politeness; or, minding your P's and Q's. In C. En Corum, T. Cedric Smith-Stark, \& A. Weiser (Eds.), Papers from the Ninth Regional Meeting of the Chicago Linguistics Society (pp. 292-305). Chicago: Departmento de Lingüística, Universidad de Chicago.

Lasarte, M. C., Sánchez Sáez, J. M., Ávila, A. M., \& Villena, J. A. (2008). El español hablado en Málaga III. Nivel de estudios superior. Málaga: Sarriá.

Lasarte, M. C. \& Villena, J. A. (2008). El español hablado en Málaga II. Nivel de estudios medio. Málaga: Sarriá.

López Morales, H. (1989). Sociolingüística. Madrid: Gredos.

Moreno Fernández, F. (1998). Principios de Sociolingüística y Sociología del Lenguaje. Barcelona: Ariel.

Puga, J. (1997). La atenuación en el castellano de Chile. Valencia: Tirant lo Blanch Libros.

Ramos, I (2005). El estilo de vida de las personas mayores y la comunicación publicitaria. Un análisis empírico [Tesis doctoral]. Universidad de Alicante.

Samper, M. (2013). La atenuación lingüística en el español de Las Palmas de Gran Canaria. Linguistica Espanola Actual, 35, 325-348.

Samper, M. (2017). Análisis sociolingüístico de la atenuación en el español de Las Palmas de Gran Canaria. Madrid: Iberoamericana/Vervuert.

Schneider, S. (2013). La atenuación gramatical y léxica. Oralia, 16, 335-356.

Vida, M. (2007). El español hablado en Málaga. Nivel de estudios bajo. Málaga: Sarriá.

Zadeh, L. (1965). Fuzzy sets. Information and Control, 8, 338-353. 